\title{
Characterization of solar multi-scaling magnetic loop interactions
}

\author{
Reinaldo R. Rosa*, Marian Karlický ${ }^{\dagger}$, Ademilson Zanandrea*, Robert A. \\ Sych $^{\ddagger}$, Hanumant S. Sawant ${ }^{\S}$ and Vinod Krishan ${ }^{\mathbb{I}}$ \\ ${ }^{*}$ Lab for Computing and Applied Mathematics, National Institute for Space Research (INPE), Sao \\ Jose dos Campos, SP, Brazil \\ ${ }^{\dagger}$ Astronomical Institute of the Academy of Sciences of the Czech Republic, CZ-25165 Ondřejov, \\ Czech Republic \\ ${ }^{* *}$ Division of Electronic Engeneering, Technological Institute of Aeronautis (ITA), Sao Jose dos \\ Campos, SP, Brazil \\ ¥Institute of Solar-Terrestrial Physics 126, Lermontov Street, Irkutsk, Russia 664033 \\ ${ }^{\S}$ Astrophysics Division (DAS) - INPE \\ IIndian Institute of Astrophysics, Bangalore-560034, India
}

\begin{abstract}
Solar magnetic loop structures can exist in a broad range of spatio-temporal scales and their mutual interactions are described by nonlinear processes of magnetic reconnection triggered as a secondary regime by a primary MHD instability. From the application of wavelet analysis on decimetric time series as a counterpart of spatio-temporal flare loop observations we derive, using Tajima's model for quasi-periodic MHD coalescence, possible ranges of minimum Sagdeev potential. From this parameter we obtain the Alfven period of nonlinear oscillation for a typical mutual solar loop interaction observed by SOHO and TRACE.
\end{abstract}

Keywords: Sun: flares - Sun: radio radiation - Sun: loop interaction - Method: spectral analysis, wavelets

PACS: $96.60 . \mathrm{Pb} ;$ 96.60.Rd; 89.75.Da

\section{INTRODUCTION}

Usually, solar flare theoretical models consider the unstable interaction of the currentcarrying loops as the primary flare process (for review see Sakai \& de Jager, 1996) where the MHD coalescence instability involves bulk current redistribution in a matter of the Alfvén time scale (Finn and Kaw, 1977; Pritchett and Wu,1979). This is a typical nonlinear process of reconnection, probably triggered as a secondary process by the primary instability (Bhattacharje et al, 1983). Based on this formalism, Tajima et al. (1987) presented a model with the nonlinear coalescence instability of interacting current loops. They used this model for the interpretation of the quasi-periodic variations of the solar flare radio emission in the microwave range. They assumed that the quasi-periodic electric field, generated during the mutual loop coalescence processes, accelerate electrons and these electrons produce the observed radio emission.

Generally not only two current loops, but multiple current structures can participate in the current loop coalescence process. Actually, there are many observations indicating the ocurrance of mutual loop interactions related to the energy release processes in the solar atmosphere and solar flares (Shimizu et al. 1992, Smartt et al. 1993, Šimberová et al. 1993, Wang et al. 2002, Fárník and Karlický 2002). In this scenario, all the 
filamentary currents can interact in the same way as described in the Tajima et al. (1987) model, but in a multi-scale process. In the solar atmospheric plasma such a mechanism can be a scaling free process, as in the case of flares and nanoflares ocurrence distribution (e.g. Wheatland (2000) and references therein) or millisecond time scales in a single flare observed in the metric and decimetric ranges (Rosa et al., 2002a), indicating that the radio observations can demonstrate a more general picture of the interacting loops.

Recently, using the Global wavelet Analysis (GWA), the authors reported the power spectra of the $3 \mathrm{GHz}$ solar radio emission, observed during the June 6, 2000 flare, with time resolution of $0.6 \mathrm{~s}$, as a $1 / f^{1.66 \pm 0.16}$ power law (Rosa et al., 2002b). This spectral index characterizes a fully developed turbulent process driving the interacting loop dynamics. However, since higher resolution data is available, it is possible to characaterize the variabilitity in terms of their persistence strengh (Malamud and Turcotte, 1999). The intermitent turbulence, a typical multi-scaling process, characterized by $1 / f^{1.66}$ can have a Wiener component (short range correlations) in higher spatio-temporal fragmentation scales. Physicaly, the wavelet decomposition periods of the radio fluctuations is a signature of the loop system nonlinear oscillations (Rosa et al, 2002b). Thus, the statistical persistence characterized from the radio time series can suggest a Wiener process component driving the loop nonlinear oscillations in the higher spatio-temporal scaling resolution.

In the following sections of the paper we provide a more detailed and complementary spectral analysis of the $3 \mathrm{GHz}$ radio flux variability considering its fine wavelet decomposition. For the first time, these time intervals are used as an input parameter in otder to obtain the Alfven period and the minimum Sagdeev potencial of a observed solar multi-scaling magnetic loop system.

\section{DATA AND WAVELET DECOMPOSTION}

\section{Data}

The June 6, 2000 flare, classified as X2.3, was observed during 15:00-17:00 UT in the active region NOAA AR 9026. A full-halo coronal mass ejection and the type II radio burst were reported in association with this flare (Solar Flare NOAA Report). During the flare, two impulsive phases at 15:14-15:40 UT and 16:34-16:40 UT were observed by the Ondřejov radiospectrograph (Jiřička et al. 1993). During the first impulsive phase the broadband pulsations and continuum were recorded, whereas during the second impulsive phase the pulses (bandwidth $\sim 800 \mathrm{MHz}$, duration $\sim 5$ s) consisting of many narrowband spikes were observed (Figure 1). The pulses seen in the spectrum correspond to the quasi-periodic peaks observed in the $3 \mathrm{GHz}$ radio flux record (upper part of Figure 1). The second phase of the flare was also observed by the EIT/SOHO and SXT/Yohkoh instruments. The EIT $195 \AA$ image at 16:36:11 UT and that of SXT/Yohkoh observed at 16:36:41 UT (Figure 2) show an arcade of parallel flare loops with brightenings between them, indicating loop-loop interactions. According to the model of Tajima et al. (1987) we interpret the $3 \mathrm{GHz}$ radio flux variations as radio manifestations of the loop interactions. 

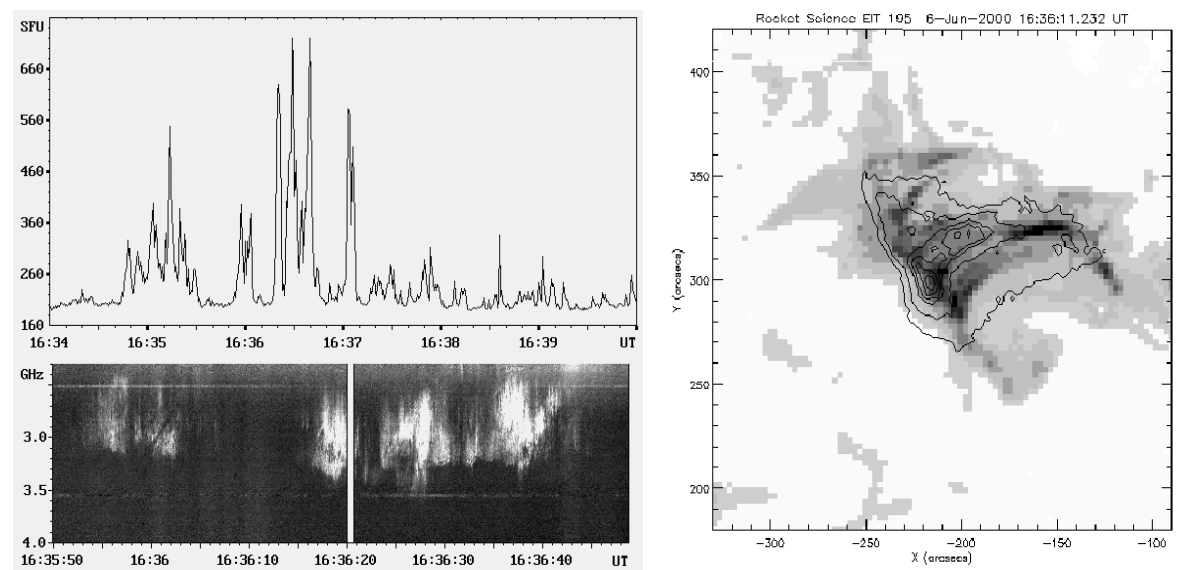

FIGURE 1. The Ondřejov $3 \mathrm{GHz}$ solar radio flux for the June 6, 2000 flare (on the rigth) and their correspondent images observed by $\mathrm{SOHO}$ and Yokoh instruments

In fact, the power-spectral density of the corresponding $3 \mathrm{GHz}$ time series variability has a power-law dependence on scale (Rosa et al 2002a, b). The power-law measures the correlations between "adjacent" values within the time series, separeted by an arbitrary scale ranging from the time series resolution to the time series duration.

In order to characterize the energy balance involved in this multi-scale plasma interacting process, we analyze the time variations of the $3 \mathrm{GHz}$ radio flux by the wavelet decomposition method. This analyses has been performed on the full time interval series of 16:34:00 to 16:40:00 UT (Figure 1 upper part) with time resolution of 0.6s (a time series composed by 600 points).

\section{Wavelet Decomposition}

The power-law from a given time series $x(t)$ measures the correlations between adjacent values separeted by a lag $\Delta t$ (beeing $\Delta t>\tau$, where $\tau$ is the time series resolution) within the time series.

The wavelet transformation of a quantitity $x(t)$ is its deconvolution into a set of functions $w_{a, b}$ where $w_{a, b}(t)=a^{-1 / 2} w\left[a^{-1}(t-b)\right]$, all derived from the mother wavelet (MW) by translation $b$ and scaling $a$ (e.g. Farge, 1992; Torrence and Compo, 1998). The MW may be chosen to best reveal the structure of the signal under consideration and for very short time scale variability under nonlinear modulation we choose the Morlet wavelet (Morlet, 1983).

The wavelet decomposition classes of the $3 \mathrm{GHz}$ time series was obtained following a dyadic scale, using one scale at each octave from 1 to 9 . The time-scale of the real part of wavelets coeficients of the respective time series were considered. This decomposition structure respectively corresponds to $(32-64 \times 0.6)$ scale limits of the band-pass decomposition filter. It can be seen that larger structures or low-frequency 


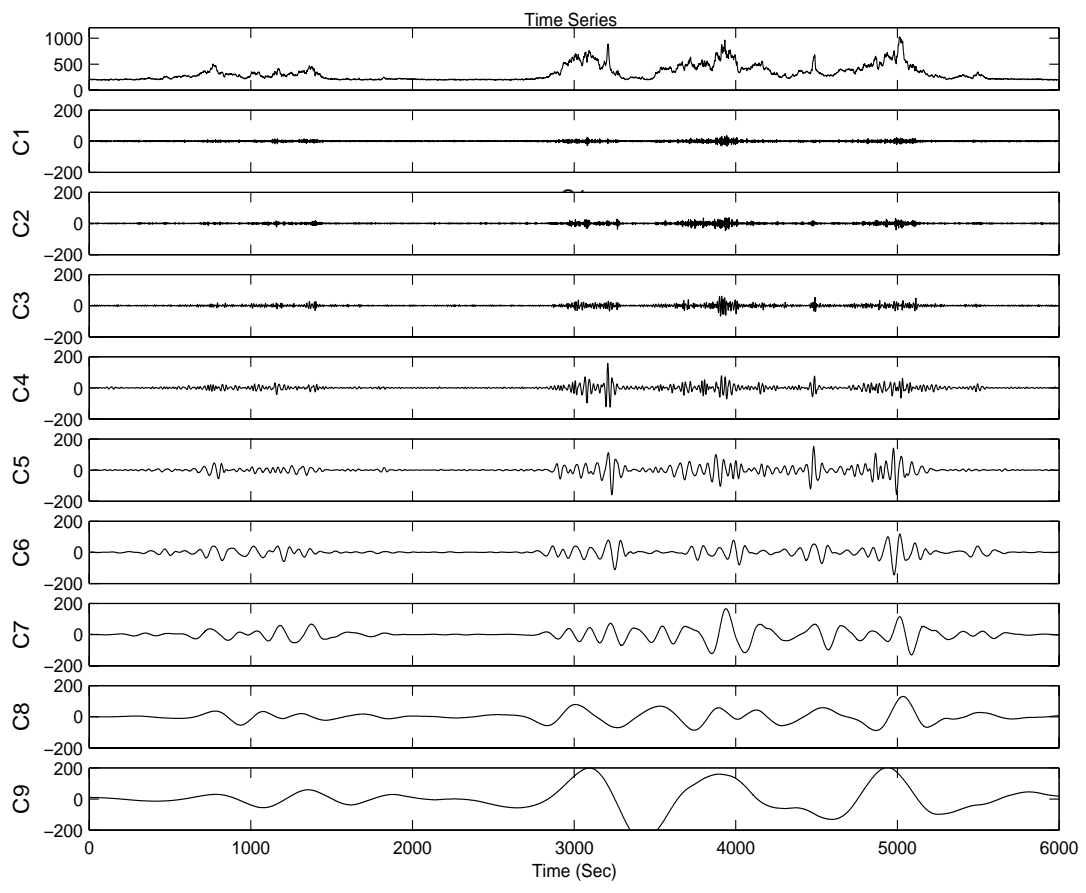

FIGURE 2. The wavelet decomposition of the time series showed in Figure 1.

package of the original signal appear in this decomposition. This analysis allows us to infer that our range of temporal variability goes from 0.6 s (given by the best time resolution of the instrument) up to $400.8 \mathrm{~s}$ (given by the lowest variability component of our decomposition) (See Table 1). A precise methodology for wavelet multiresolution signal decomposition is given by Mallat (1989).

It is worth noticing that in the wavelet decomposition all decomposed signals have the same wavelet spectral index $\left(\beta_{w}\right)$, so that they are self-similar in a log-log slope. Threfore, the result shown in Figure 3 and Table 1 also confirm that wavelet analysis is a powerful tool to investigate stochastic signals and short lived structures (Percival and Walden, 2000).

\section{THE MUTUAL LOOP OSCILLATION AND ITS MINIMUM SAGDEEV POTENTIAL}

The interacting current loops model (Tajima et al. 1987) take into account the nonlinear coalescence instability of interacting current loops, where the quasi-periodic regime of these processes is given by the characteristic period $T_{O S}$ of nonlinear oscillations: 
TABLE 1. Decomposition Period (DP)

Decomposition

Class

\begin{tabular}{rr}
$\mathbf{D P}(\mathbf{s e c})$ & \\
\hline $\mathrm{C} 1$ & 0.6 \\
$\mathrm{C} 2$ & 1.4 \\
$\mathrm{C} 3$ & 4.6 \\
$\mathrm{C} 4$ & 8.8 \\
$\mathrm{C} 5$ & 26.4 \\
$\mathrm{C} 6$ & 48.4 \\
$\mathrm{C} 7$ & 86.8 \\
$\mathrm{C} 8$ & 260.5 \\
$\mathrm{C} 9$ & 400.8 \\
\hline
\end{tabular}

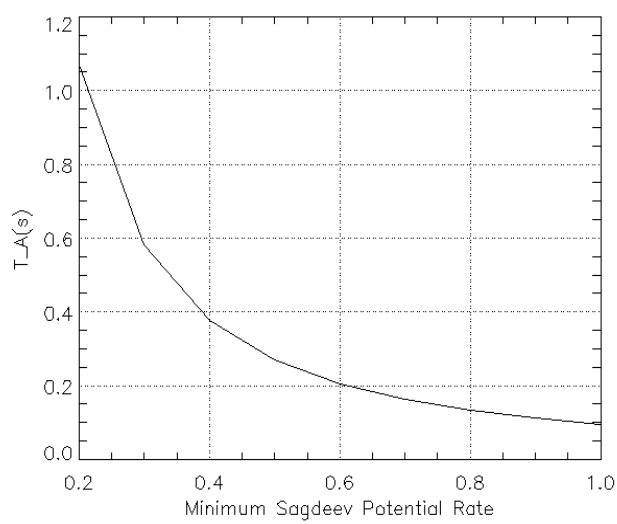

FIGURE 3. The Alfven period as function of the minimum Sagdeev potential rate.

$$
T_{O S}=2 \pi(-2 E)^{-3 / 2} T_{A}^{-2},
$$

where $E$ is the initial "energy" of the system, $T_{A}$ is the Alfvén period $\left(\lambda / v_{A}\right.$ with $\lambda$ beeing the magnetic field scale length and $v_{A}$ is the Alfvén speed).

The period $T_{O S}$ becomes longer when $E$ tends to zero and tends to a minimum value $T_{\text {min }}$ when $E$ tends to the minimum Sagdeev potential $-(1 / 2) T_{A}^{-2} \gamma$ :

$$
T_{\min }=2 \pi \gamma^{3 / 2} T_{A}
$$

where $\gamma$ is the minimum Sagdeev potential rate: the ratio between the kinetic and magnetic energy densities involved in the energetic oscillating multi-loop interaction. 
From the multilevel wavelet decomposition one obtain the $T_{\min }$ and the characteristic timescales corresponding to $T_{O S}$. The first value is taking as input parameter for the calculation of $T_{A} \times \gamma$ as given by the equation 2 that can be write as:

$$
T_{A}(\gamma)=\left(T_{\min } / 2 \pi\right) \gamma^{-3 / 2}
$$

while the values for $T_{O S}$ (Table1) are taked as input parameters for the computation of $E$ as a function of $T_{O S}$ and $T_{A}$ :

$$
E\left(T_{O S}, T_{A}\right)=(\gamma)=-(1 / 2) T_{A}^{-4 / 3}(1 / 2 \pi)^{-2 / 3} T_{O S}^{-2 / 3}
$$

In Figure 4, the nonlinear behavior of Alfven period is shown as a function of the minimum Sagdeev potential rate. The convergence of the Alfven period gives an almost constant period of 0.1 seconds when more than $80 \%$ of the magnetic energy is transformed into the kinetic nonlinear oscillating energy. From equation 4 is it possible to show that the total energy of the system is related to the nonlinear oscillations only into the range of $T_{A}<0.5 \mathrm{~s}$.

\section{CONCLUDING REMARKS}

It can be seen that the wavelet analysis decomposition offers a fine-grain characterization of the multi-scaling flare loop interactions in the solar atmospheric plasma. In particular, the wavelet analysis of the $3 \mathrm{GHz}$ radio flare observations combined with EIT/SOHO $195 \AA$ and SXT/Yohkoh images have demonstrated a more general picture of the solar interacting loops, as a cluster of spatio-temporal scales. The loop interaction scales can be ranging from very small to very large spatio-temporal scales. In the pure time domain one can say this range goes from, at least, 0.6 s up to $\sim 8$ hours time scales. Physically, it can be interpreted as a scaling free and a self-correlated intermmitent stochastic process. This implies that there is a bunch of many parallel interacting current loops and each specific loop may again consist of a bunch of the filamentary sub-currents. In such a system all the filamentary currents can interact as a multi-scale process and the intermittent turbulence of overlapping plasma resonance can lead to the onset and evolution of MHD coalescence instability. This stochastic behaviour of coherent plasma structures can undergo complex changes as the nonlinear dynamic system evolves, similar to those commonly observed in equilibrium phase transitions.

In some rare cases it may happen that there exists a dominant scale to generate a specific time period of the oscillations. The presence or absence of specific periods is also a function of the spatio-temporal resolution of the observations, indicating a self-similar fragmentation of the released energy. Based on the Tajima's mutual loop interaction model, we found that for small values of the Alfven period involved, with a upper limit of $0.5 \mathrm{~s}$, more than $80 \%$ of the magnetic energy must be transformed into the kinetic nonlinear oscillating energy. 


\section{ACKNOWLEDGMENTS}

The authors are grateful to the Brazilian funding agency FAPESP under grants 00/10043-9 and 03/06665-2. M.K., V.K. and R.A.S. thanks FAPESP authorities for supporting their visit to INPE. This work was also supported by the grants A3003202 and S1003006, AS CR and from Russian Foundation for Basic Research ( ${ }^{o}$ 01-02-16290) and INTAS 543.

\section{REFERENCES}

.Amaral, L. A. N., Goldberger, A.L., Ivanov, P.C. \& H. E. Stanley, H.E. 1998, Phys. Rev. Letters $81(11), 2388$

.Arneodo, A., Bacry, E., Muzy, J.F. 1995, Physica A 213,232

.Bhattacharjee, A.; Brunel, F.; Tajima, T. 1983, Physics of Fluids Volume 26 (11),3332

.Fargé, M. 1992, Ann. Rev. Fluid Mech. 24,395

.Fárník, F. \& Karlický, M. 2002, Yohkoh10 Meeting, Kona, Hawaii, 128

.Finn, J. M.; Kaw, P. K. 1977, Physics of Fluids 20,72

.Jiřička, K., Karlický, M., Kepka, O., \& Tlamicha, A. 1993, Solar Phys. 147,203

.Mallat, S. 1989, IEEE Pattern Anal. and Machine Intell.11(7),674

.Mandelbrot, B. B. 1999, Multifractals and 1/f noise, Springer-Verlag, New York

.Morlet, J. 1983, Sampling Theory and Wave Propagation. NATO ASI, vol. F1, Issues on Acoustic Signal/Image Processing and Recognition (C.H. Chen ed.). Springer-Verlag

.Percival, D., Walden, A.T. 2000, Wavelet Methods for Time Series Analysis, Cambridge Univ. Press, UK, Cambridge

.Prichett, P. L.; Wu, C. C. 1979, Physics of Fluids 22,2140

.Rodrigues Neto, C., Rosa, R.R., Ramos, F.M., Zanandrea, A. 1999, Asymmetry and Self-similarity in the Wavelet Spectrum, in SPIE Conference on Wavelet Applications in Signal and Image Processing VII 3813,914

.Rosa, R.R., Vats, H.O., Ramos, F.M., Zanandrea, A., Neto, C.R., Fernandes, F.C.R., Bolzan, M.J.A., Rempel, E.L., Brito, R.C., Vijaykumar, N.L., Sawant, H.S. 2002a, Adv. Space Res. 29(3),463

.Rosa, R.R., Ramos, F.M., Sawant, H.S., Fernandes, F.C.R., Vijaykumar, N.L., Zanandrea, A., Karlický, M. 2002b, Proc. 10th European Solar Physics Meeting, ESA SP-506, 737

.Sakai, J. \& deJager, C. 1996, Space Sci Rev 77,1

.Smartt, D.F., Zang, Z., \& Smutko, M.F. 1993, Solar Phys. 148,139

.Shimizu, T., Tsuneta, T., Acton, L.W., Lemen, J.R., \& Uchida, Y. 1992, Publ. Astron. Soc. Japan 44,L147

.Šimberoví, S., Karlický, M., \& Švestka, Z. 1993, Solar Phys. 146,343

.Tajima, T., Sakai, J., Nakajima, N., Kosugi, T., Brunel, F., Kundu, M.R. 1987, ApJ 321,1031

.Torrence, C., Compo, G.P. 1998, Bulletin of the American Meteorological Society, 79(1),61 
Copyright of AIP Conference Proceedings is the property of American Institute of Physics. The copyright in an individual article may be maintained by the author in certain cases. Content may not be copied or emailed to multiple sites or posted to a listserv without the copyright holder's express written permission. However, users may print, download, or email articles for individual use. 